استخدام طريقتي المقاومية الكهريائية والجذبية في استكشاف وتحديد الجيولوجيا تحت السطحية في جزء من منطقة اسكي موصل / شمال غرب العرلق فيله فيله

$$
\begin{aligned}
& \text { أيمن محمود أحمد منيف محجوب المحجوب بثار عزيز محمود }
\end{aligned}
$$

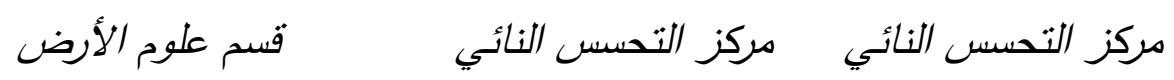

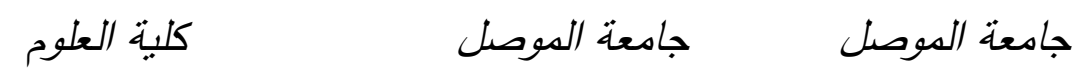

$$
\begin{aligned}
& \text { جامعة الموصل }
\end{aligned}
$$$$
\text { (تاريخ الاستلام 2007/9/6 ، تاريخ القبول 2007/11/21) }
$$

الملخص (المنام

يتضمن البحث الحالي تطبيق طريقتي المقاومية الكهربائية والجذبية لاستكثاف وتحديد الجيولوجيا

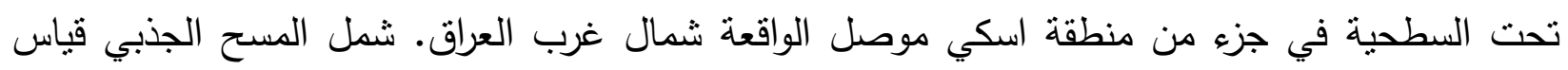

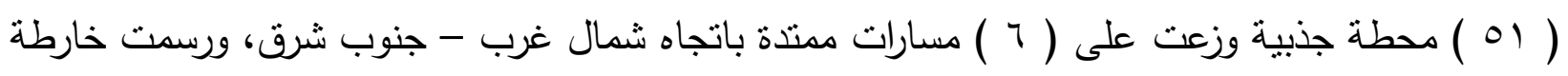
شذوذ بوجير .

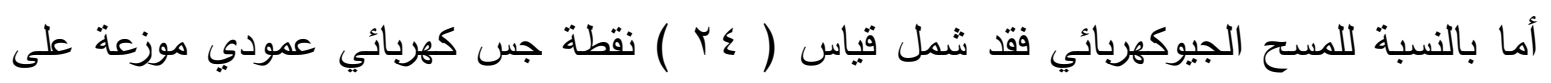
( ) ( ) مسارات ممتدة باتجاه المسارات الجذبية اعلاه، وتم تقسير جميع نقاط المسح الجيوكهربائي تفسيرا كميا

$$
\text { باستخدام برامج الحاسوب الجاهزة. }
$$

تم الاعتماد على نتائج المسح الجيوكهربائي فقط في تحديد الجيولوجيا تحت السطحية لمنطقة الدراسة.

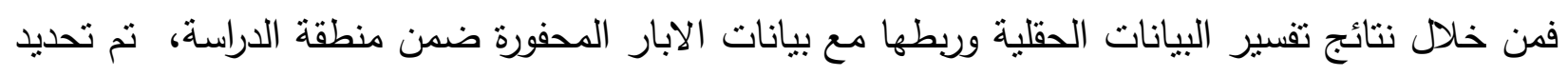
التتابعات الطباقية تحت السطحية في المنطقة والتي تبين انها تعود للعضو الاسفل من تكوين الفتحة. أيضا

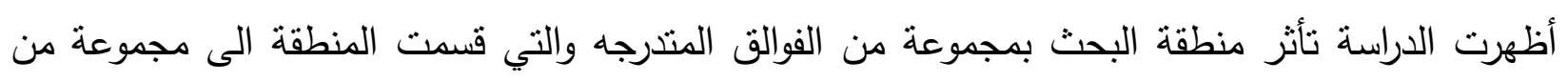
البلوكات الصاعدة والهابطة. 


\title{
The Using of the Resistivity and Gravity Geophysical Methods in the Detection of the Subsurface Geology in Asky Mosul Area / North West of Iraq
}

\author{
Ayman M. Ahmed \\ Remote Sensing \\ Center \\ Mosul University
}

\author{
Muneef M. Al-Mahjoob \\ Remote Sensing \\ Center \\ Mosul University
}

\author{
Bashar A. Mahmoud \\ Deptartment of Geology \\ Collage of Sciences \\ Mosul University
}

\begin{abstract}
Present research includes application of the methods of electrical resistivity and gravity to explore and determine the subsurface geology in a part of the Asky Mosul area situated to the north-west of Iraq. The gravity survey included (51) stations distributed along (6) traverses extended towards the northwest-southeast, and drew Bouguer anomaly map.

As for the geoelectrical survey has included measurement of (24) vertical electrical sounding points distributed along (4) traverses in the same direction of the gravity traverses above. All the (VES) points are interpreted quantitatively using the (IPI 2.1 Win) software.

Through the interpretation of the geoelectrical results and link them with the boreholes sequences within the study area, we identified the subsurface stratigraphical sequences in the study area which appeared as the lower member of the Al-Fatha formation. The study also showed that the area is affected by set of step faults which divided the area to some horst and grabben structures.

$$
\begin{aligned}
& \text { المقدمة } \\
& \text { هناك عدة دراسات جيوفيزيائية أجريت في المنطقة وماحولها، وتم من خلالها تحديد التعاقبات }
\end{aligned}
$$

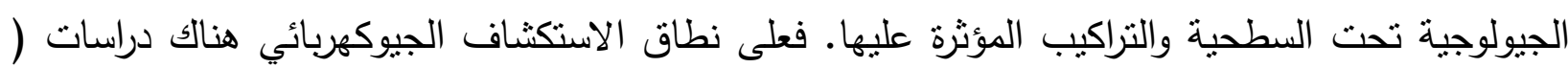

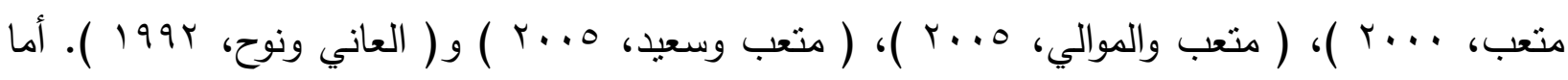

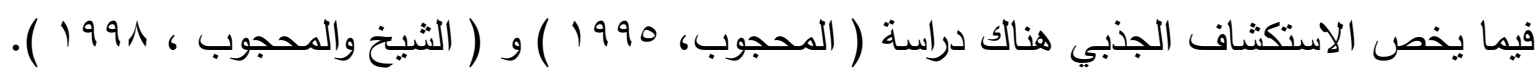

$$
\begin{aligned}
& \text { تقع منطقة الدراسة شمال العراق على بعد حوالي ( •7 ) كم شمال غرب مدينة الموصل والى الجنوب }
\end{aligned}
$$

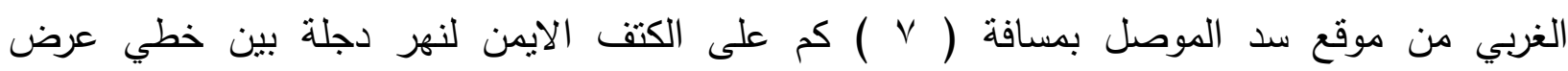

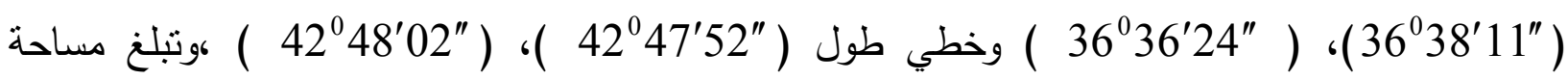

$$
\begin{aligned}
& \text { منطقة الدراسة ( ع ) كم ك، الثكل ( ) ( ). }
\end{aligned}
$$


يهدف البحث الحالي الكثف عن التتابعات الطباقية في جزء من منطقة اسكي موصل ومدى نأثر هذه التتابعات بالتراكيب الجيولوجية تحت السطحية.

\section{جيولوجية منطقة الدراسة}

تكتونيا تقع منطقة الدراسة في النطاق الثانوي بطمة - جمجمال ضمن نطاق أقدام الجبال من الرف

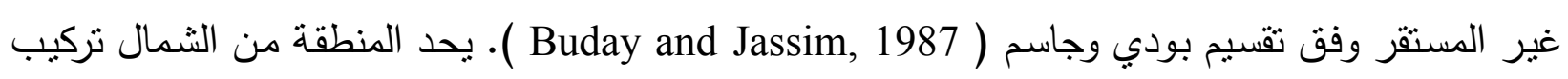

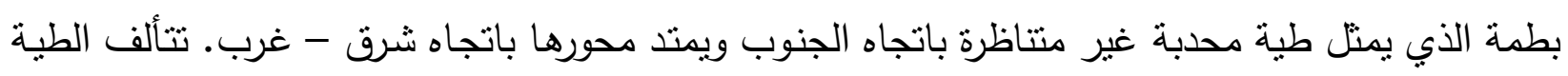

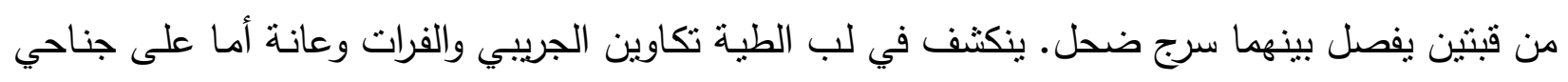

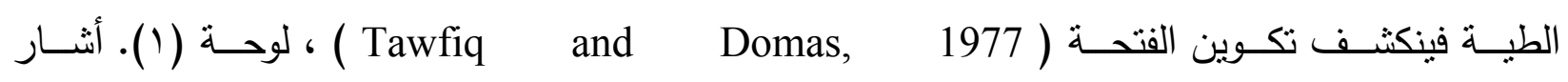

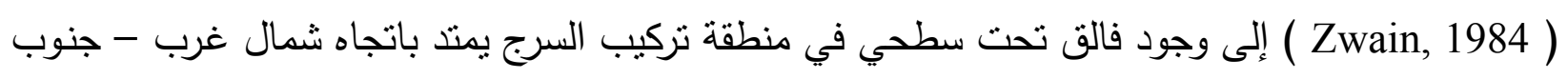

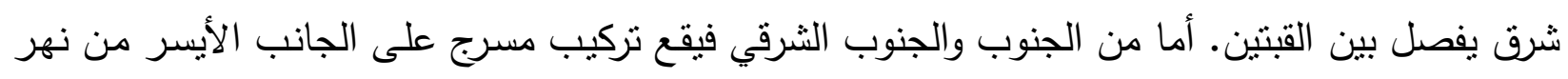

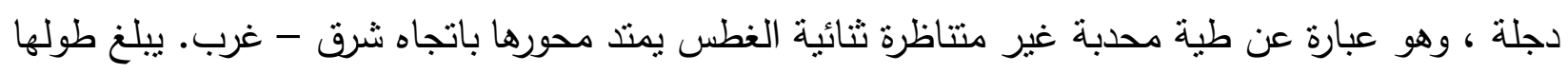

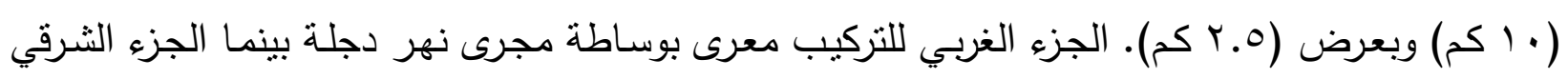

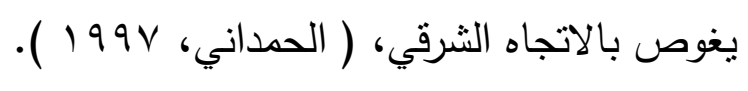

طباقيا، ينكثف تكوين الفتحة (Al-Fatha) في عموم منطقة الدراسة، لوحة (1). من خلال ملاحظة

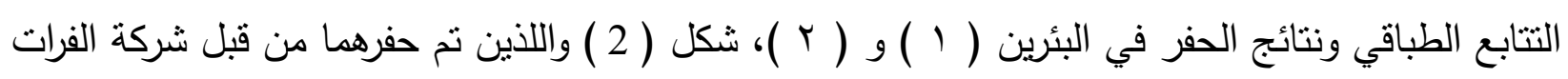

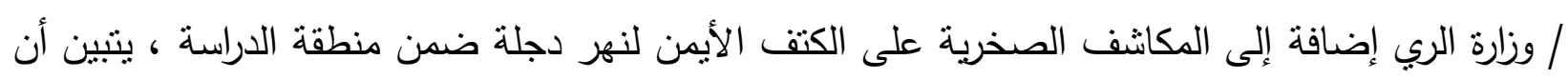

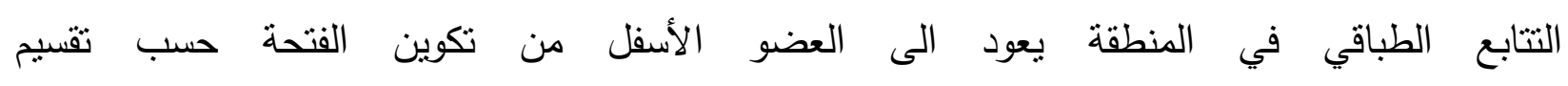
.( Tawfiq and Domas, 1977 ) 


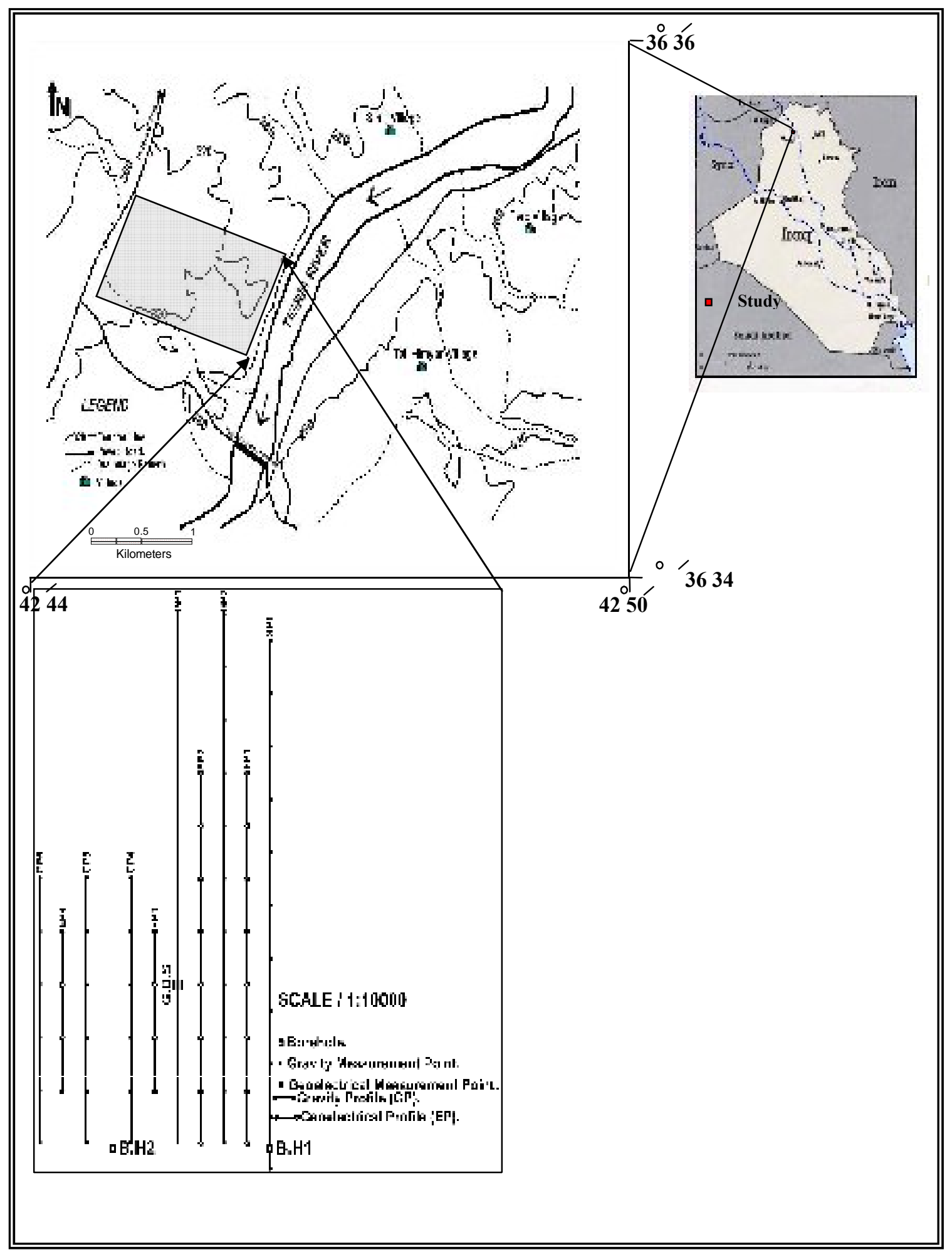

الثكل I : خارطة موقع منطقة الدراسه موضح عليها نقاط القياس. 


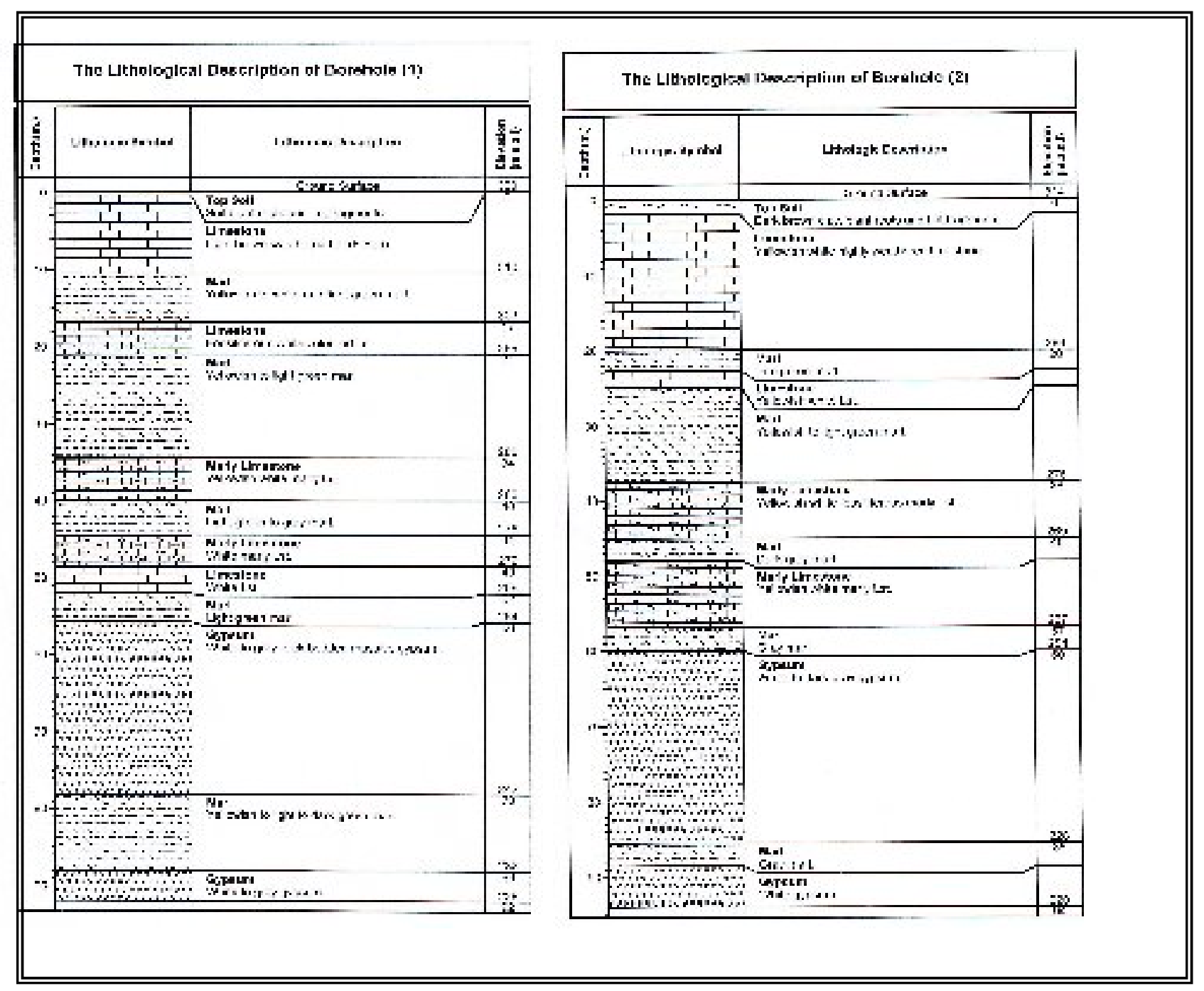

الثكل r : التتابع الطباقي للآبار ( ( و r ) .

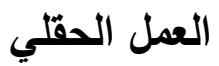

تضمن العمل الحقلي اجراء مسحين جذبي و جيوكهربائي لمنطقة الدراسة وهما جزء من مسح جيوفيزيائي عام تضمن كلتا الطريقتين انجز من قبل دائرة التحريات الجيوتكنيكية التابعة لشركة الفرات

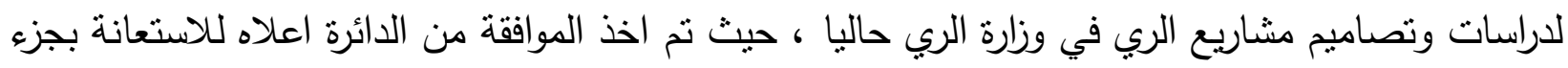
من بيانات المسح الجيوفيزيائي لانجاز هذا البحثي.

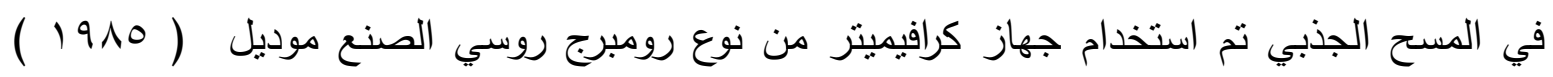

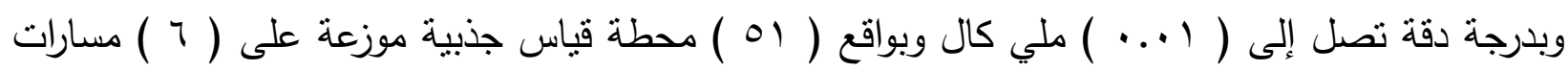

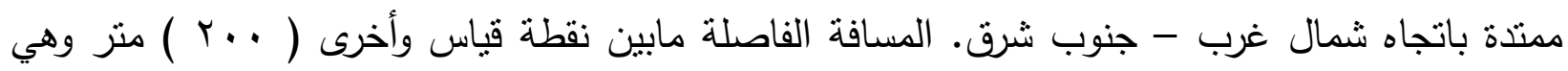

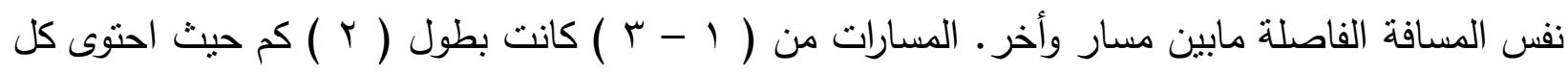
منها على ( 11 ) نقطة قياس والمسارات من ( ع - 7 ) بطول ( 1 ( ) كم ويحوي كل منها على ( 1 ( ) نقاط 


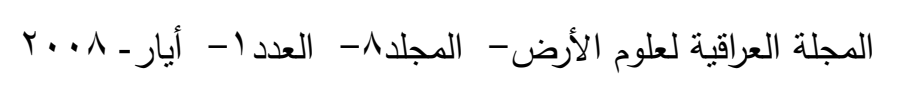

قياس. اختيرت المحطة ( ع ) ضمن المسار الثالث كمطة مرجعية للقياسات الجذبية حيث تم تصحيح جميع القراءات الجذبية نسبة لهذه المحطة ، الثنكل ( 1 ( ).

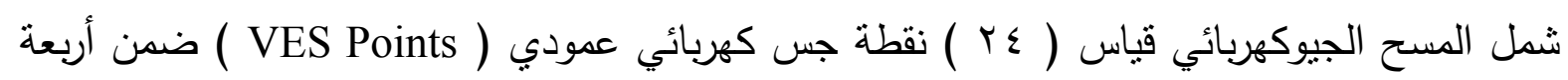

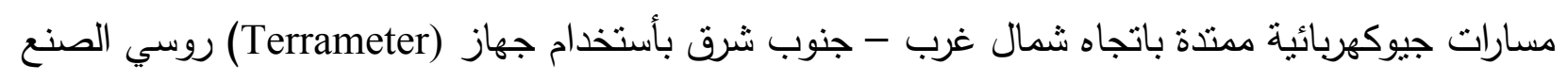

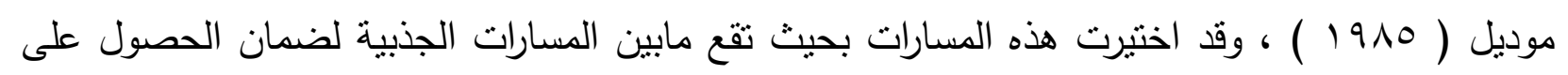

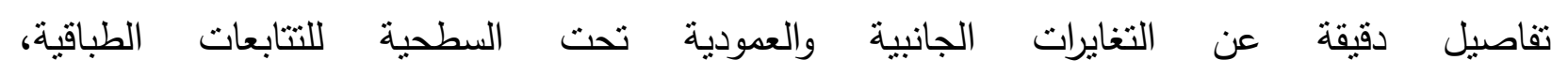

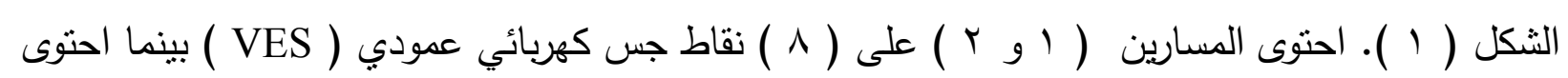

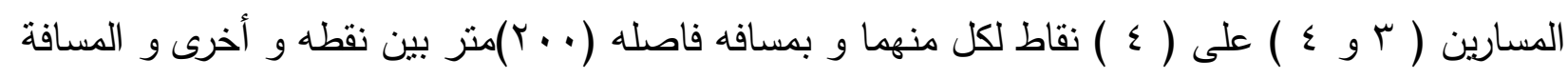

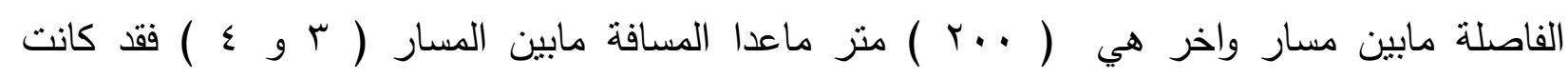

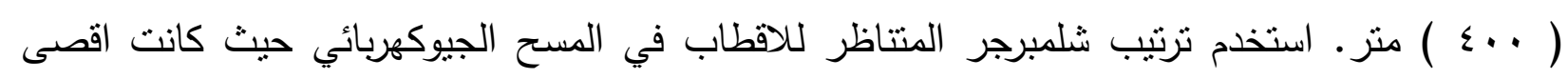

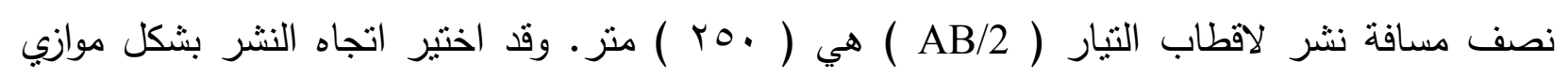

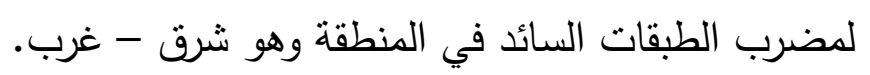

\section{تفسيرالمعلومات الحقلية}

أ- تفسير معطيات المسح الجيوكهربائي: ان منحنيات الجس الكهربائي العمودي يمكن تفسيرها وصفيا بالاعتماد على انكالها او كميا باستخدام

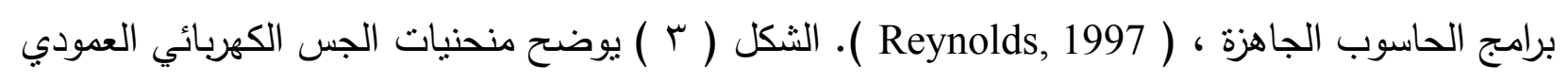
حيث تبين ان معظمها من نوع ( KH ) والذي يعكس وجود اربعة انطقة جيوكهربائية عامة في منطقة

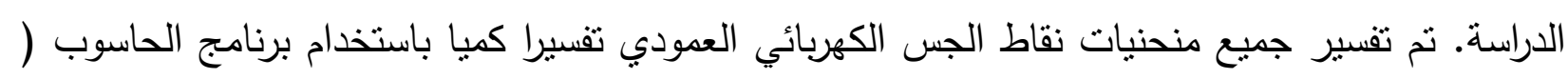
(IPI 2.1 win, 2001 المستحصلة من التفسير الكمي لنقاط الجس. تم رسم اربعة مقاطع جيوكهبائية رئيسية ضمن منطقة الدراسة وعلى وفق مسارات المسح الجيوكهربائي

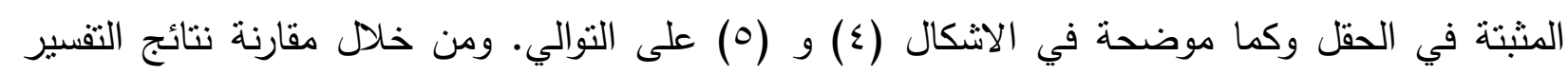

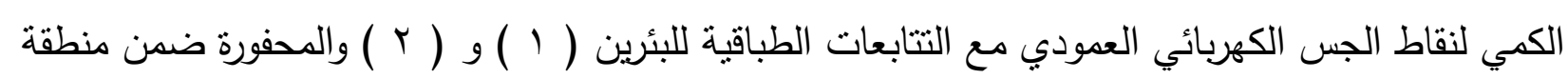

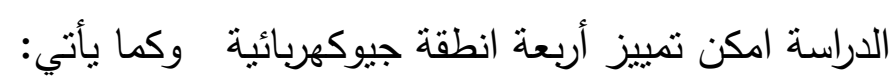

النطاق الاول: تتراوح قيم المقاومية النوعية الكهربائية لهذا النطاق (1.1 - . . (1) اوم.متر

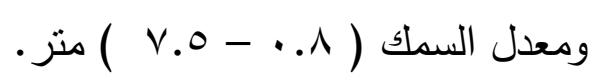

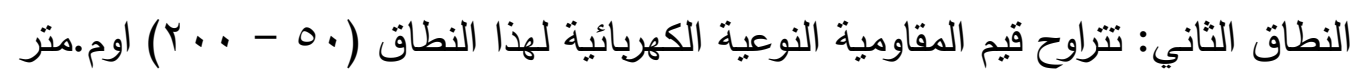

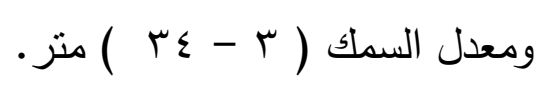


النطاق الثالث: تتراوح قيم المقاومية النوعية الكهربائية لهذا النطاق (• (1 - .0) اوم.متر ومعدل السمك

$$
\text { • (9) - } 1 . \Lambda \text { ) }
$$

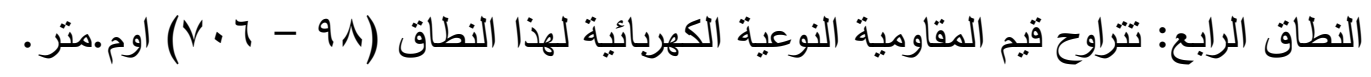

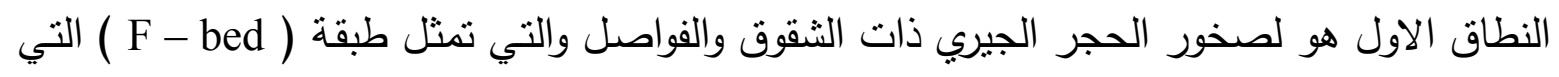
تقصل مابين العضوين العلوي والسفلي لتكوين الفتحة حسب تقسيم(Tawfiq and Domas, 1977 ). النطاق الثاني يمثل صخور الحجر الجيري المارلي. اما النطاق الثالث فيمنل صخور المارل ذات الثقات المقاومية الواطئة، وبالانتقال الى النطاق الرابع لوحظ ظهور مقاوميات نوعية كهربائية عالية منلت صخور الجبس.

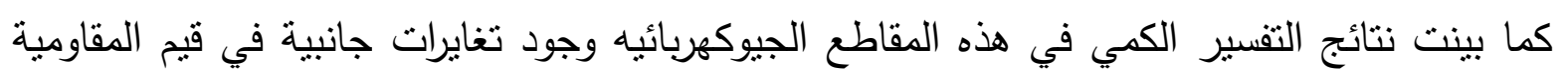

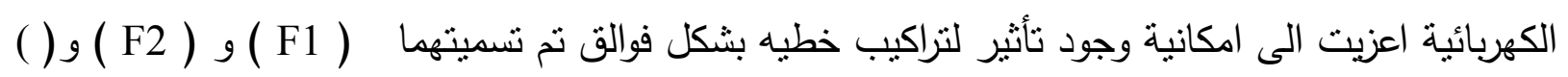

F3 الثكلين ( 4 ( ) و ( 5 ).

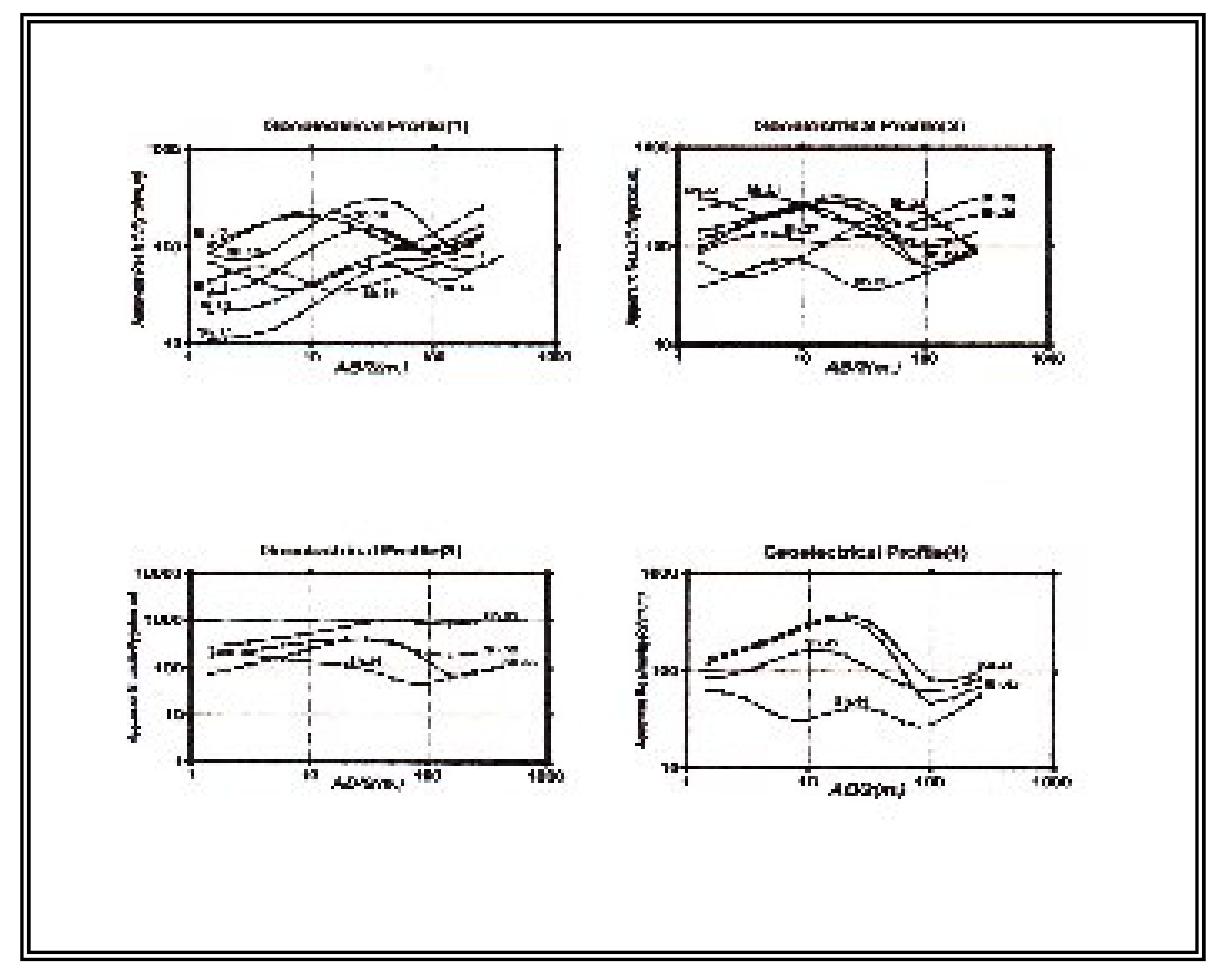

الثكل 3 : المنحنيات الحقليه لنقاط الجس الكهربائى العمودي.

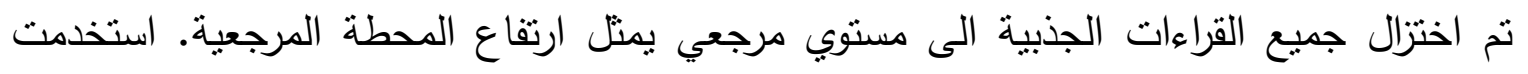

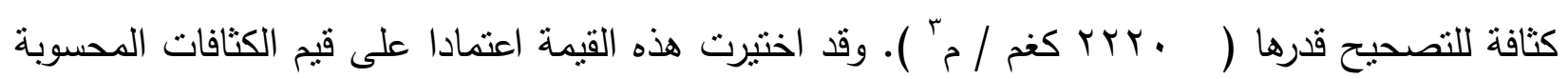

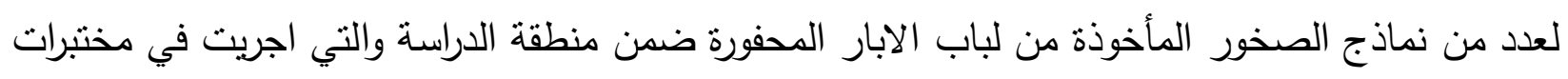

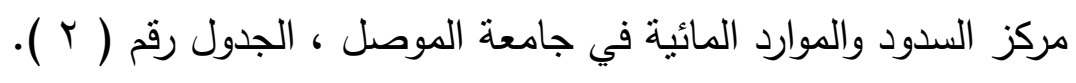




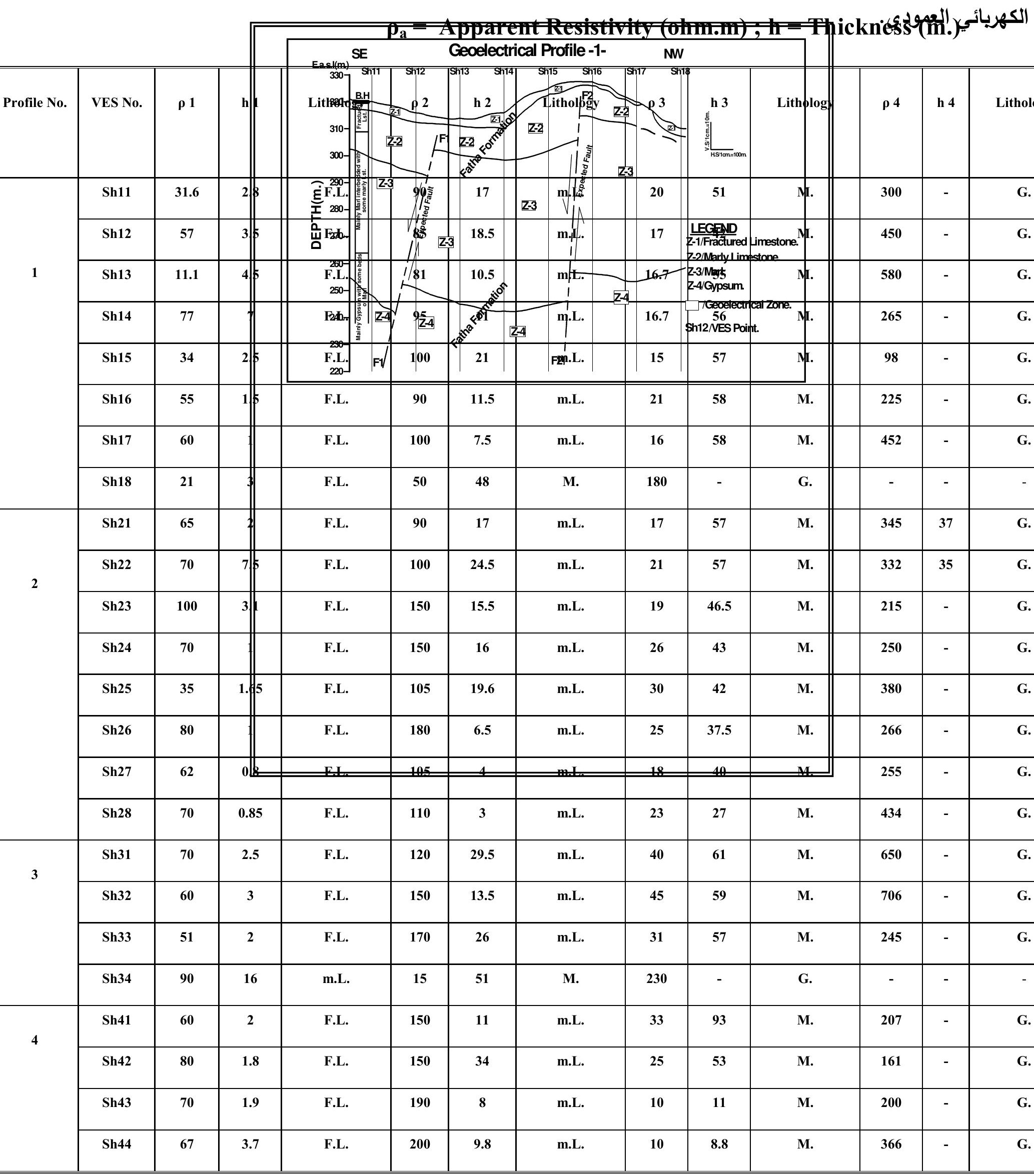




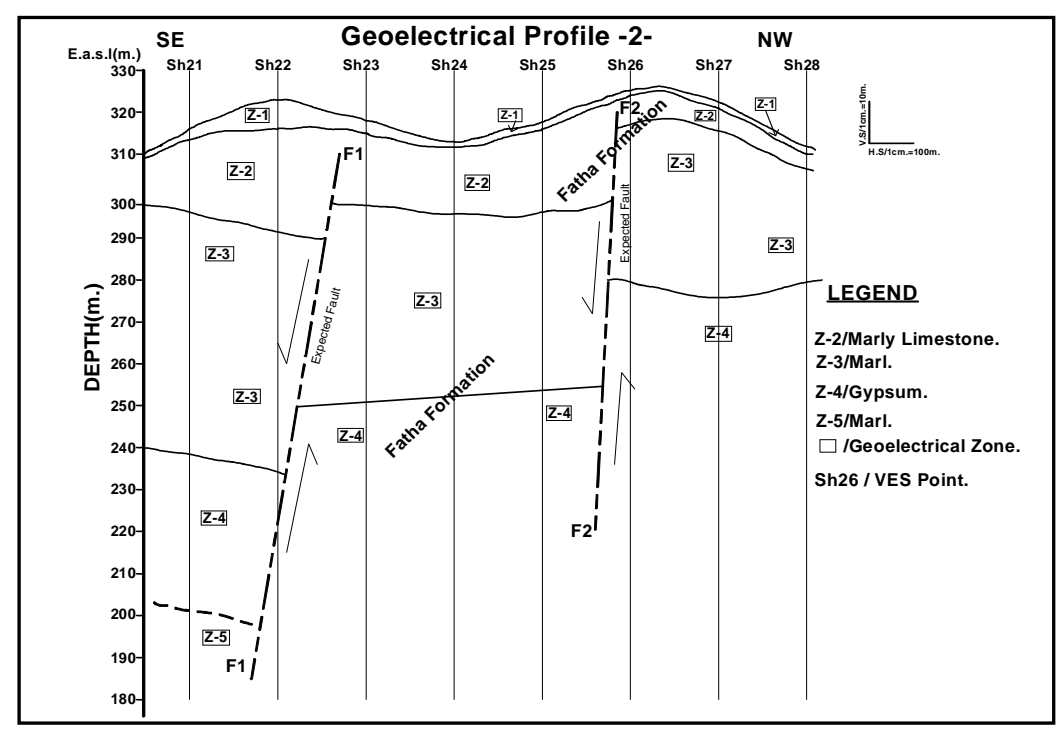

\section{الثكل4 : المقاطع الجيوكهربائيه ( ا و r ).}

تم رسم خارطة كنتورية لنذوذ بوجير الملاحظ وبفاصلة كنتورية قدرها( 0.5 ) ملي كال ، النشل ( 7 ). لوحظ على خارطة الثذوذ الجذبي وجود شواذ طولية موجبة وسالبة تمثد باتجاهات شمال - جنوب و شرق - غرب. الا ان قيم هذه الشواذ كانت ضئيلة واحيانا لاتتجاوز ( ه. . ) ملي كال ، لذلك لايمكن الاعتماد على هذه النتائج في تحديد الجيولوجيا تحت السطحية لمنطقة الدراسة.

الجدول ب: قيم الكثافات المعدلية لصخور منطقة الدراسة.

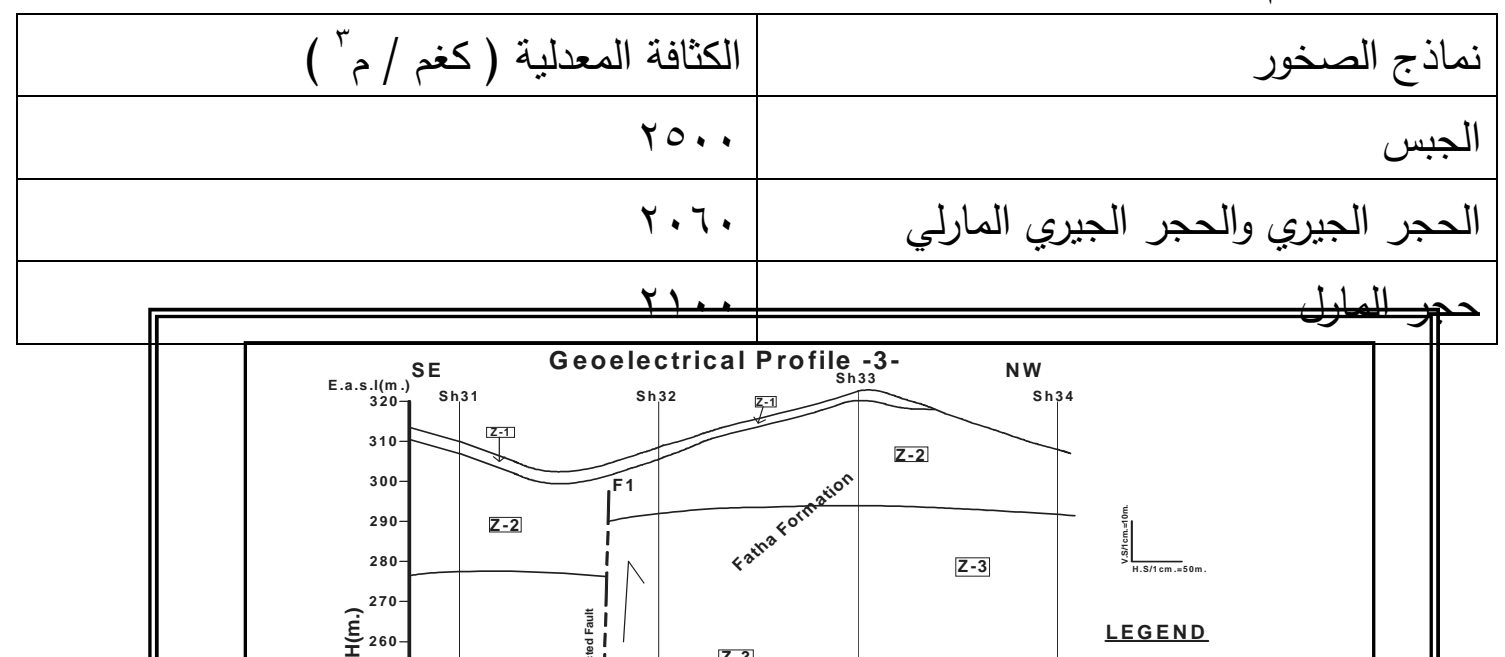




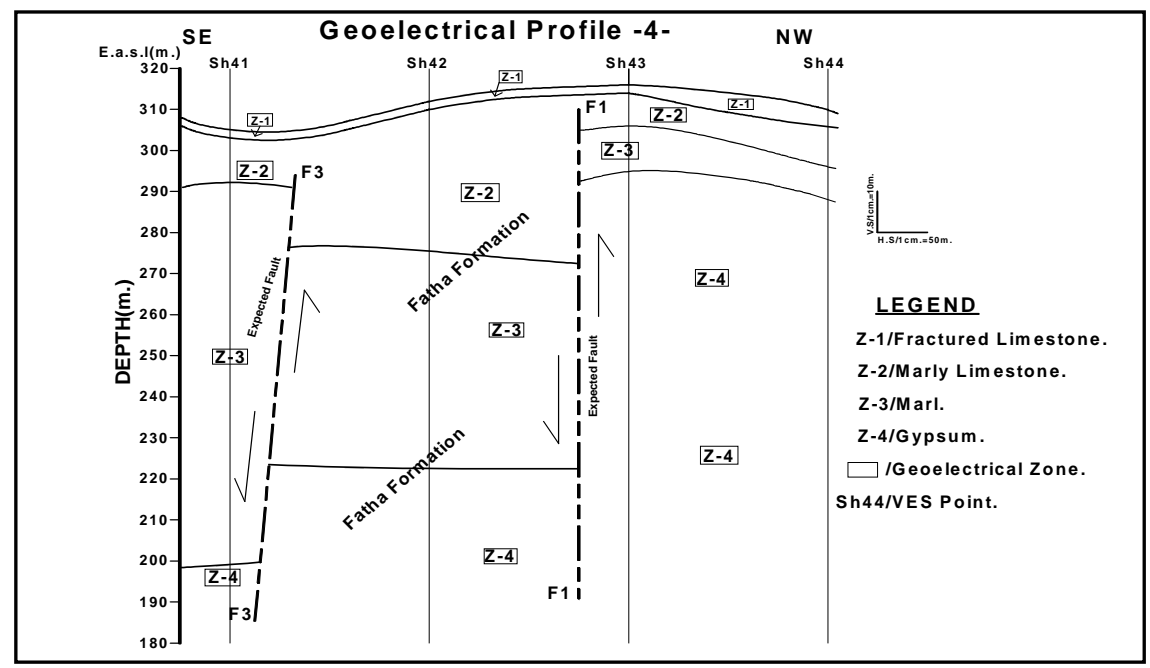

الشكل 5 : المقاطع الجيوكهربائيه (س و ؟ ).

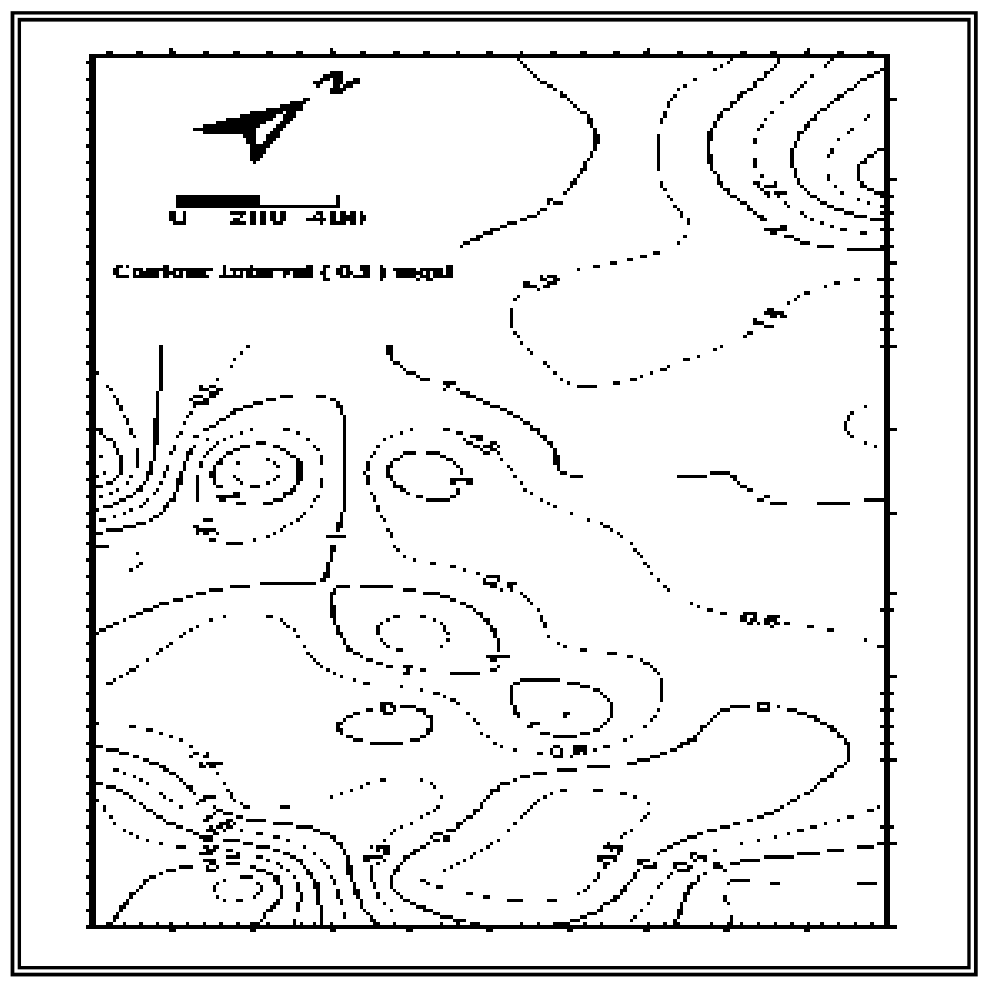

الثكل 7: خارطة شذوذ بوجير لمنطقة الدراسة. 


\section{المناقشتة والاستتتاجات}

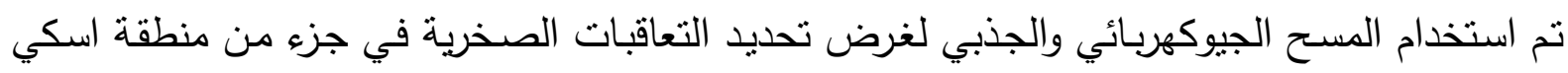

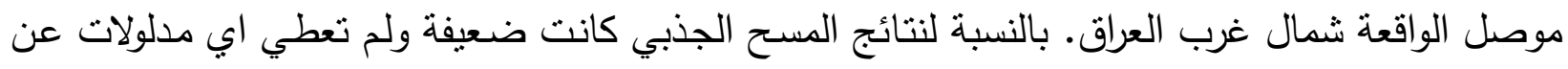

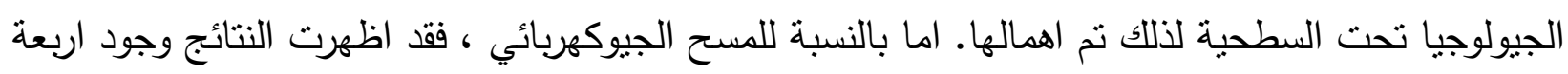

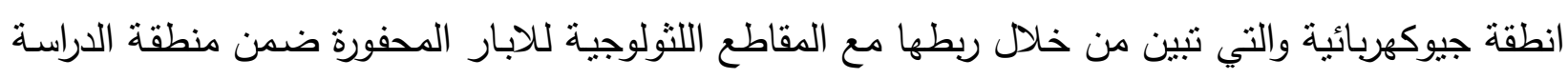

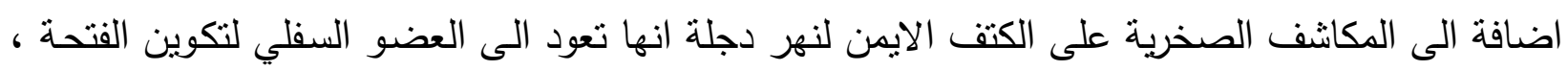

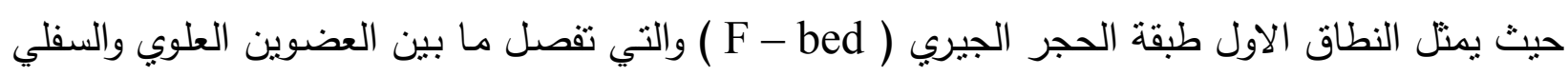
لتكوين الفتحة حسب تقسيم ( Tawfiq and Domas, 1977 ).

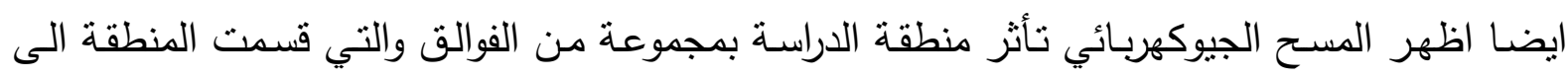

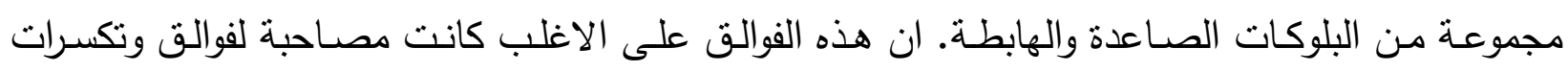

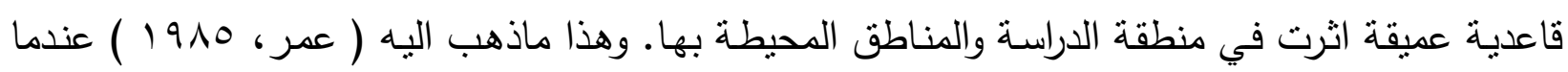

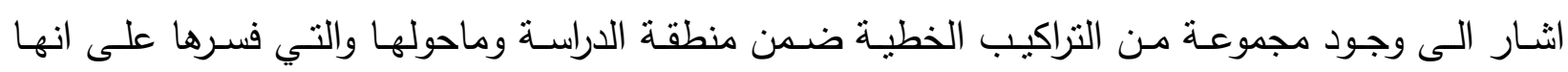
انعكاسات لفوالق تحت سطحية عميقة ، اضافة الى الفالق الرئيسي الذي يفصل بين تركيبي بطمة الثرقية الثرائ وبطمة الغربية والمثبت من قبل ( Zwain, 1984 ) والذب يعتقد ان امتداده يؤثر ضمن منطقة الدراسة الحالية.

\section{المصادر العربية}

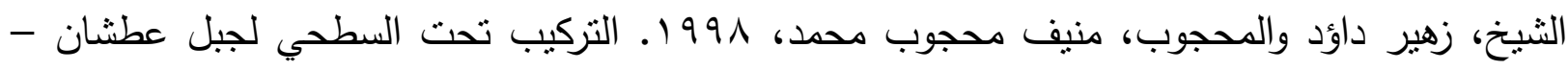

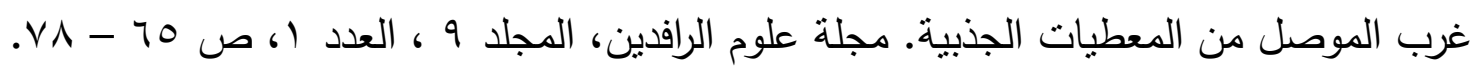
الحمداني، عادل علي بلال، 1997. تطور مقطع في نهر دجلة مابين سد الموصل ومدينة الموصل.

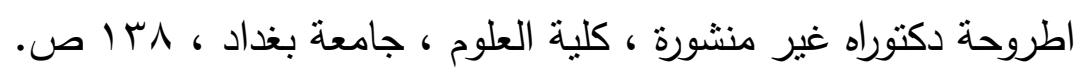

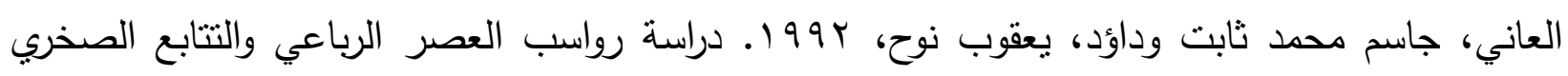

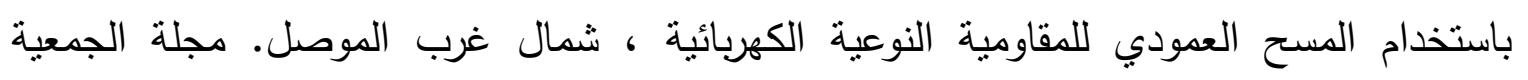

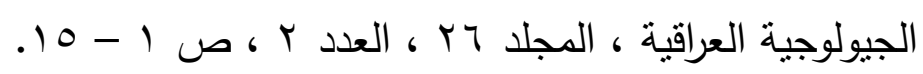

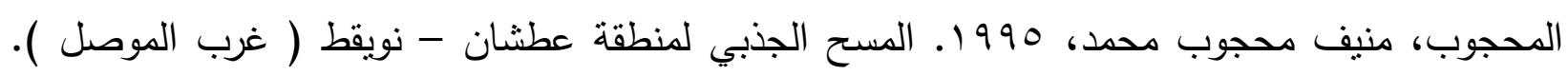

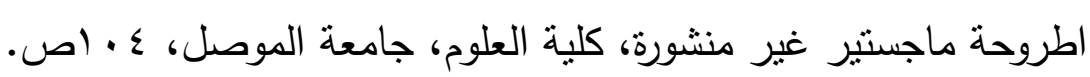

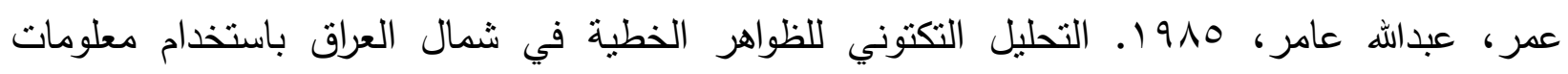

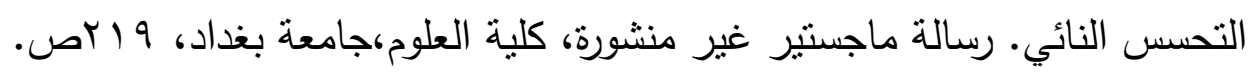

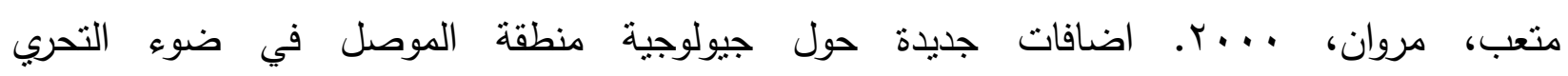

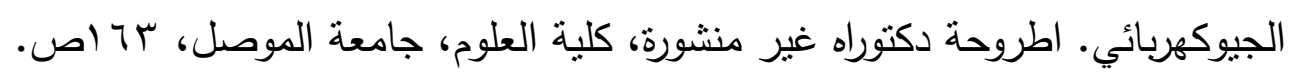


متعب، مروان والثيخ، زهير داؤد، ب . . . . دراسة جيوكهربائية استطلاعية لمنخفض الموصل. المجلة

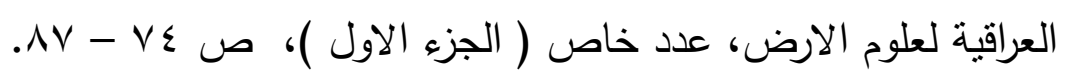

متعب، مروان والموالي، علي سعد الدين، ه .. . . دراسة جيولوجية لسهل علان الجنوبي، شمال

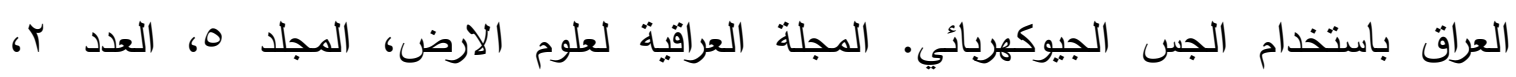

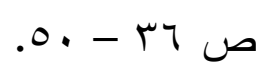

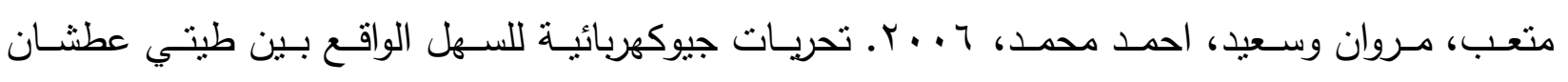

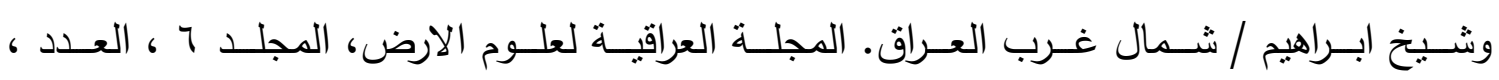

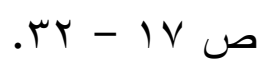

\section{المصادر الاجنبية}

Buday, T. and Jassim, S. Z., 1987. The regional geology of Iraq. Tectonism, Magmatism and Metamorphism, vol. 2, D.G. of Geological Survey and Mineral Invistigation, Baghdad.

IPI, 2, 2001. Win. Version 2.1, Moscow State University, Dept. of Geophysics.

Reynolds, J. M., 1997. An Introduction to applied and environmental geophysics.

John Wiley and Sons, U.K., 796 P.

Tawfiq, J. M. and Domas, J., 1977. Regional geological mapping of Duhok - Ain Zala area. Unpub. Report, SOM, Iraq.

Zwain, J. A., 1984. The structural analysis of geological fracture traces in Mushorah - Ain Zalah area, Northern Iraq. Journal of the geological society of Iraq, vol. 17, pp. 25-51. 


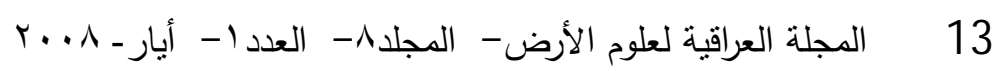

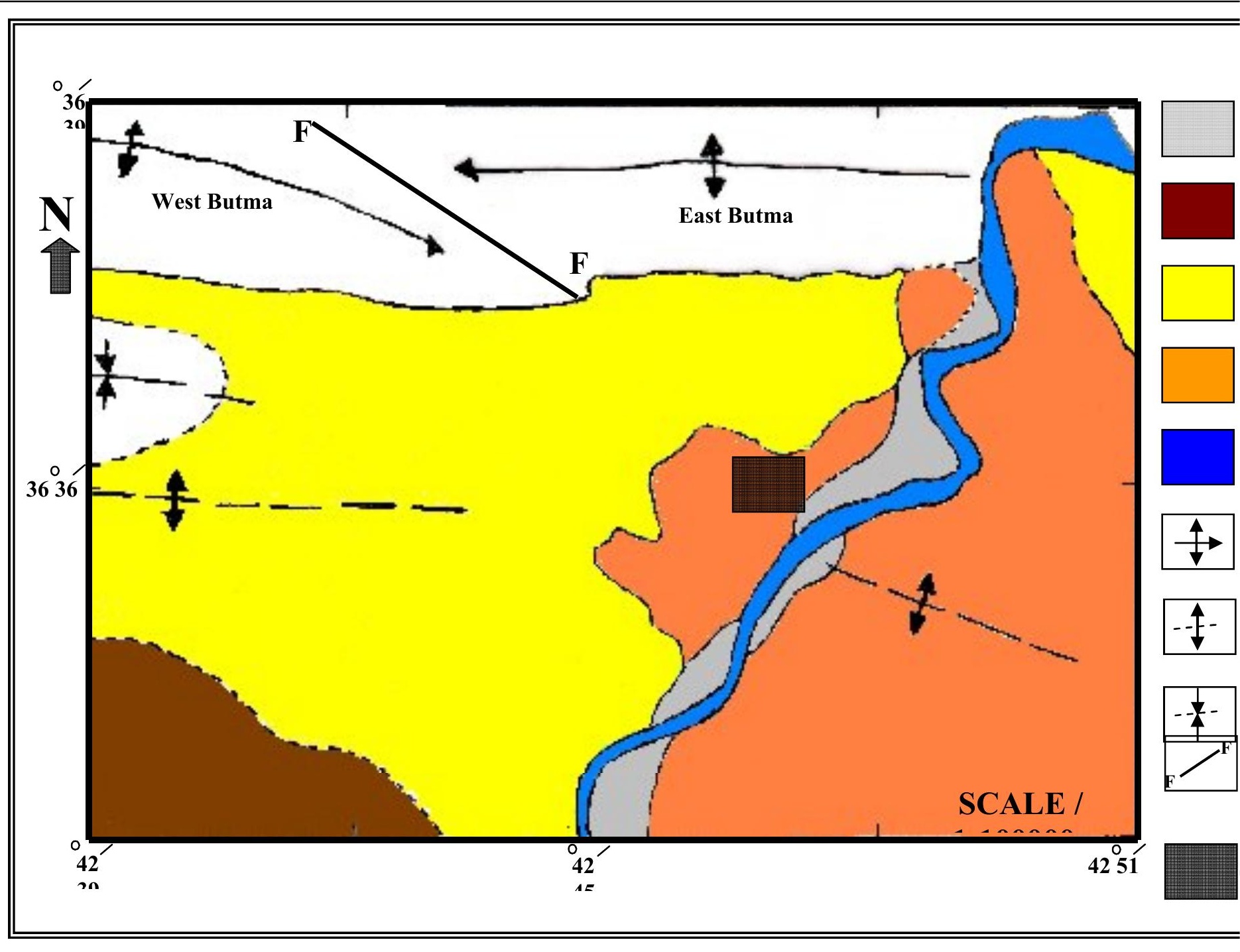

